\title{
A Classification of Leprosy Foot Deformities
}

\author{
W. M. I.ENNOX, B.s(:., F.R.(:.s.(EN(i.), F.R.(:.S.(EDIN.)
}

Department of Orthopaedies, Christian Medical College and Hospitals, Vellore, S. India, Lepra Fellow C.M.C. Hospital, Vellore

(iLAWSON and SEDDON (Ig60), in a study of the late sequelac of sciatic palsy found that of the patients who suffered from persistent ulceration, less than half exhibited anaesthesia, but all had some fixed deformity of their feet or toes. 'They concluded that fixed deformity was a more important cause of ulceration than loss of sensibility. In the leprosy patient, an undeformed anaesthetic foot can be kept frec from ulceration by the wearing of appropriate shoes. Deformed anaesthetic feet, on the other hand, exhibit a high incidence of ulceration even with footwear, particularly if the deformity concentrates weight bearing onto a small area of skin.

Observation of a large number of foot patients has revealed certain repetitive patterns of deformity, and has enabled a relatively simple classification to be drawn up. It is the purpose of this paper to describe a classification which may provide a useful basis for description and discussion of an apparently complex spectrum of deformity.

It is usual to classify leprosy deformities as either primary or secondary. Primary deformities are those due to the disease process itself. They include loss of eycbrows and all paralytic deformities, such as are unavoidable unless the case comes under adequate medical treatment early. Secondary deformities are duc to causes other than leprosy itself, and are preventable. 'They usually result from septic complications affecting an anaesthetic extremity. 'This concept is retained in the present classification, and it should be stressed that most secondary deformities in the foot result directly or indirectly from trophic ulceration. This ulceration frequently is a result of neglect of a primary deformity.

FI ( ; . I

\section{Natural History of the Deformed Leprosy Foot}

$\begin{array}{llll}\text { Leprosy } & \text { Primary } & \text { Secondary } & \text { Destruction } \\ \text { Neuritis } & \text { Deformity } & \text { Deformity } & \text { (Amputation) } \\ & \text { (and Ulcers) } & \text { (and Ulcers) } & \end{array}$

All established foot deformities are associated with a tendency to ulcerate at a specific site. Without surgical intervention, such feet eventually undergo total destruction. The natural history of the untreated deformed foot is summarized in Fig. (I). No worker who has witnessed this sequence of events, can fail to agree with the conclusion reached by CILAWSON and SEDDON.

Figure (2) outlines the proposed classification. The direct relationship between Groups I and II is indicated by arrows, and the site of specific ulceration of each group is also shown. 
FI G . 2

\section{Primary Deformity}

I Muscle Imbalance

Sile of Ulcer

A I)ropped Foot

Antero-lateral Border

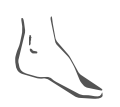

B Dropped Foot

Antero-medial Border

(Perone Intact)

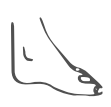

C Claw 'loes

1)orsum and Pulp of l'oes

Metatarsal Heads

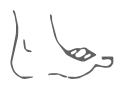

\section{Secondary Deformity}

II Fixed Deformities

Shortened Equinus

Foot

Inverted Foot:

Destruction Lateral

Ray

Shortened Foot with

Destruction of Medial

Ray

Shortened Plantigrade

Foot

I I I

Deformity due to Joint

Neuropathy

IV

Heel Deformities

V

Calcaneus Deformity
Site of Ulcers

Anterior Border

Lateral Border

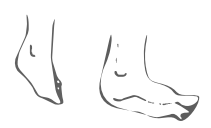

Anterior Border

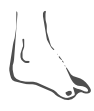

Anterior Border
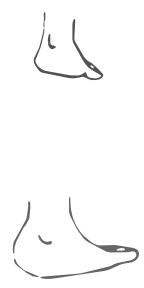

Heel: Multiple Sinuses

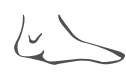

Heel

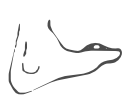

GROUPS I AND II

Dynamic deformities resulting from muscle imbalance: Fixed deformities

(a) Dropped foot, due to lateral popliteal paralysis, results in a high stepping gait in which the foot is violently slapped onto the ground at each step. The antero-lateral border bears the brunt of the impact because unopposed action of the tibialis posterior causes a slight varus 
imbalance. It is here that the ulcers associated with the deformity occur. Bone and tissue is shown in the destruction of the lateral ray and shortening of the foot follows, and as the deformity becomes fixed, it passes into Group II.

(b) Most examples of foot drop are complete, but in some instances the peronei are spared. With strong peronei and absent tibialis anterior the lateral balance of the foot is upset in favour of the evertors. 'The pressures along the antero-medial border are accentuated, and ulcers typically occur here, with destruction of the medial bones. If the peronei later became paralysed, and the foot is first seen when the deformity has become fixed, one may be left wondering why a dropped foot sometimes exhibits this atypical pattern of destruction.

(c) Clawing of the toes is the result of paralysis of the intrinsic muscles of the foot supplied by the posterior tibial nerve. Hyperextension of the metacarpophalangeal joints and flexion of the interphalangeal joints brings the tips of the pulps into contact with the ground, where blisters and ulcers occur, and osteomyclitis of the terminal phalanx. At a later stage, the metacarpophalangeal capsule stretches and the base of the proximal phalanx subluxates dorsally, and ultimately a complete dorsal dislocation occurs, with the acutely clawed toes overriding the heads of the metatarsals. Once subluxation occurs, the toes tend to become stiff in their clawed position, and the deformity passes into Group II. The effects of subluxation are:

(I) 'The toes are lifted clear of the ground, removing the risk of tip ulceration.

(2) 'The pressure accepted by the toc pulps during 'push off' in walking cannot now be taken, and passes further back to the metatarsal heads. 'The extensor digitorum longus tendon shortens and the joint capsule develops contracture.

(3) The fibro-fatty pad (part of the specifically differentiated plantar fascia) underlying the metatarsal head is drawn upwards from beneath the metatarsal heads and thinned out beneath them. 'The metatarsal heads thus become relatively subcutaneous and subject unprotected skin to high pressure and shear stress during walking. Ulceration occurs under them with a high risk of osteomyelitis. The most characteristic sequel is destruction of the metatarsal heads with shortening of the foot, but the subluxed toes are spared, and remain perched along the anterior margin of the foot.

Group I deformities are passively correctible, but they pass into Group II when the deformity becomes fixed by contracture of the tendoAchilles, joint capsules, and fibrosis of subcutaneous tissues. X-rays of Group I deformities show a normal foot skeleton, except when a claw toe deformity is seen. In Group II, bone and joint changes are apparent, notably concentric atrophy or absence of the distal parts of the metatarsals. Rarefaction of bone indicates active bone inflammation, and illustrates the mode of evolution of the deformity. 
The plantar surface in Group II deformities is frequently reduced in area, but is usually free from scars (except at the anterior or lateral margin). 'The plantar surface is preserved because it is protected from weight bearing; this is an important fact from the point of view of treatment.

\section{G R OUP II I}

\section{Deformity due to bone and joint neuropathy}

Any bone or joint in the foot may undergo destruction as a result of continued function after injury in the absence of pain. 'This aspect of leprosy deformity is not well documented, but a forthcoming contribution (BRAND and HARRIS) is likely to prove helpful. In the meantime, attention should be drawn to certain clinical features of the condition, and to a not uncommon deformity resulting from it. 'The foot is swollen and warm, and exhibits excessive subtalar or midtarsal movement, with crepitus. The sole is convex, with flattening of the medial arch, and palpable descent of the head of the talus and navicular. 'There is contracture of the tendo-Achilles, which draws the posterior tuberosity of the calcancum backwards and upwards, and contributes to dorsal hinging of the foot at the mid-tarsal plane. A pressure area develops in the centre of the sole or in the instep, and it is here that the ulcers associated with this deformity appear. Descriptively, the deformity has been named 'Boat Foot'. By means of the warmth and swelling, the process may be distinguished from osteomyclitis and cellulitis. X-ray shows crumbling and fragmentation of one or more bones, with roughening of joint surfaces and irregular sclerosis. 'These appearances may also be attributed to old septic arthritis, but the absence of sinus scars is helpful in making the distinction.

\section{Heel Deformities}

\section{GROUP IV}

These constitute a separate surgical problem, and are therefore put into a group of their own. Most cases result from ulcers under the heel. 'The primary ulcer has a particularly obscure aetiology, but once deformity of the calcaneum has occurred, it is perpetuated by localized high pressures, or by rupture of an unyielding scar by shear stresses. The spectrum of deformity in this group ranges from erosions and spurs on the undersurface of the calcaneum, to bizarre deformities resulting from partial or total destruction of the talus, and calcaneum. These patients may have no heel at all, and may be transmitting weight direct from the lower end of the tibia through scarred skin to the ground.

Destruction of these bones may result from osteomyelitis, septic arthritis of the subtalar joint, neuropathic disintegration, or a combination of these processes. Clinically, the heel is scarred, the plantar pulp may be destroyed and multiple sinuses may be present. X-rays show calcancal irregularities, or partial or complete loss of talus and/or the calcancum (Fig. 2). 


\section{Calcaneus Deformity}

'This deformity also poses special problems of treatment, and merits a category of its own. It results occasionally from muscle imbalance following foot drop surgery, or to avulsion fracture of the insertion of the tendoachilles. It has also been seen in calf weakness following poliomyelitis in patients who have also contracted leprosy. It carries with it a high risk of heel ulceration. On X-ray the foot skeleton may be relatively normal, or there may be absorption and flattening of the posterior tuberosity of the calcancum.

\section{DISGUSSION}

'This classification embraces the majority of leprosy foot mutilations, but in some instances a combined type of deformity may be scen, for example, a bizarre hecl deformity, and a fixed claw toe deformity with shortening of the metatarsals.

\section{S U M M A R Y}

A descriptive classification of leprosy foot deformities is outlined, and the close relationship of deformity to trophic ulceration is stressed. No discussion of management is attempted, but the clinical features of each group are summarized, and their relationships are described.

\section{ACK NOWLEDGEMENTS}

I a m very grateful to MR. B. V. VENKATESAN for typing services, to MR. CARL BRASS for the drawings and MR. SIGAMONEY for preparation of the illustrations.

\section{Bibliography}

ci.awson, 1. K., and stidion, H. J. The Late Consequences of Sciatic Nerve Injury. Journal of Bone and .Joint Surgery, 42-13, $1960,213$.

branis, P. w., and unkris, J. Patterns of Disintegration of the Tarsus in the Anaesthetic Foot. In course of publication. 